\title{
Phase reconstruction using fast binary 4D STEM data $\odot$
}

Cite as: Appl. Phys. Lett. 116, 124101 (2020); https://doi.org/10.1063/1.5143213

Submitted: 20 December 2019 . Accepted: 10 February 2020 . Published Online: 23 March 2020

(iD C. M. O'Leary, (D) C. S. Allen, (D) C. Huang, (D) J. S. Kim, (D) E. Liberti, (D) P. D. Nellist, and (D) A. I. Kirkland

\section{COLLECTIONS}

F This paper was selected as Featured
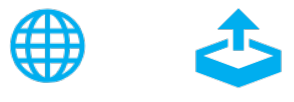

\section{ARTICLES YOU MAY BE INTERESTED IN}

Strain mapping at nanometer resolution using advanced nano-beam electron diffraction Applied Physics Letters 106, 253107 (2015); https://doi.org/10.1063/1.4922994

Subsampled STEM-ptychography

Applied Physics Letters 113, 033104 (2018); https://doi.org/10.1063/1.5040496

Detectors-The ongoing revolution in scanning transmission electron microscopy and why this important to material characterization

APL Materials 8, 110901 (2020); https://doi.org/10.1063/5.0026992

\section{Challenge us.}

What are your needs for periodic signal detection?

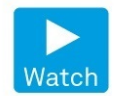

- Zurich

Instruments

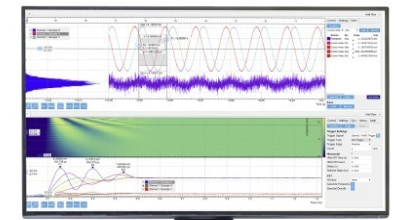

- 


\title{
Phase reconstruction using fast binary 4D STEM data 8
}

\author{
Cite as: Appl. Phys. Lett. 116, 124101 (2020); doi: 10.1063/1.5143213 \\ Submitted: 20 December 2019 . Accepted: 10 February 2020 . \\ Published Online: 23 March 2020
}

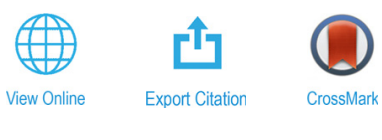

\author{
C. M. O'Leary,, (D) C. S. Allen, ${ }^{1,2}$ (D) C. Huang, ${ }^{1,2}$ (D) J. S. Kim, ${ }^{1,2,3}$ (D) E. Liberti, ${ }^{1,2}$ (D) P. D. Nellist, ${ }^{1}$ \\ and A. I. Kirkland ${ }^{1,2,3}$ (D)
}

\author{
AFFILIATIONS \\ ${ }^{1}$ Department of Materials, University of Oxford, Oxford OX1 3PH, United Kingdom \\ ${ }^{2}$ electron Physical Science Imaging Centre (ePSIC), Diamond Light Source, Didcot OX11 ODE, United Kingdom \\ ${ }^{3}$ The Rosalind Franklin Institute, Harwell Campus, Didcot OX11 OFA, United Kingdom
}

${ }^{a)}$ Author to whom correspondence should be addressed: colum.oleary@materials.ox.ac.uk

\begin{abstract}
We report the application of focused probe ptychography using binary 4D datasets obtained using scanning transmission electron microscopy (STEM). Modern fast pixelated detectors have enabled imaging of individual convergent beam electron diffraction patterns in a STEM raster scan at frame rates in the range of $1000-8000 \mathrm{~Hz}$ using conventional counting modes. Changing the bit depth of a counting detector, such that only values of 0 or 1 can be recorded at each pixel, allows one to decrease the dwell time and increase the frame rate to $12.5 \mathrm{kHz}$, reducing the electron exposure of the sample for a given beam current. Atomically resolved phase contrast of an aluminosilicate zeolite (ZSM-5) is observed from sparse diffraction patterns with isolated individual electrons, demonstrating the potential of binary ptychography as a low-dose 4D STEM technique.
\end{abstract}

Published under license by AIP Publishing. https://doi.org/10.1063/1.5143213

Transmission electron microscopy (TEM) and scanning TEM (STEM) are capable of imaging materials at atomic resolution, providing invaluable insights into their structure. Many systems, including soft (organic) materials and functional materials such as lithium-ion battery components, ${ }^{1}$ hybrid perovskites, ${ }^{2,3}$ and metal-organic frameworks, ${ }^{4}$ contain low atomic number ( $\mathrm{Z}$ ) elements and are easily damaged by the incident electron beam. The weaker scattering of low- $Z$ materials also leads to a low contrast in images, a problem that is compounded by the limited electron dose (fluence) that can be used to form the image, resulting in a low signal-to-noise ratio (SNR). Electron scattering by low-Z elements primarily leads to a small phase shift of the transmitted wave, and imaging them requires a form of efficient phase-contrast imaging. In conventional TEM, phase plates are increasingly used to enhance phase contrast. ${ }^{5,6}$

For beam-resistant samples, the STEM has been widely used to provide atomic resolution imaging and spectroscopy. ${ }^{7}$ In a STEM, the electron beam is focused to form a small probe that is scanned in a raster across the sample. Signals arising from the detection of scattered electrons can be plotted as a function of probe position to form an image. In particular, an annular dark-field (ADF) detector collects relatively high angles of scattering, offering an incoherent imaging mode whose contrast is highly dependent on the atomic number. The incoherent nature also leads to a simple imaging mechanism where the spatial resolution is controlled by the intensity distribution of the illuminating probe. However, the Z-contrast nature of $\mathrm{ADF}$ imaging makes it poorly suited to light element imaging, and the collection of only high-angle scattering means that only a small fraction $(\sim 10 \%)$ of the transmitted electrons is detected. By the principle of reciprocity, the STEM can be used for phase-contrast imaging, using a small detector that integrates only the coherent signal from the central regions of the bright-field (BF) disk for each probe position and so again uses only a fraction of the incident electrons.

Phase techniques such as differential phase-contrast (DPC), integrated DPC, ${ }^{9}$ ptychography, ${ }^{10-12}$ and annular bright-field (ABF) imaging $^{13}$ have significantly improved the SNR of STEM imaging for weak-phase-objects. As shown by Seki et al., ${ }^{14}$ ptychography offers the highest SNR of these techniques. It also has the advantage of allowing the correction of residual aberrations following acquisition, removing the need to finely focus the probe before data acquisition, and hence reducing sample exposure to the electron beam. Low dose imaging has been demonstrated for ptychography using a defocused probe that spreads the electron flux over a substantial illumination area. ${ }^{15}$ Here, we address low dose methods for ptychography with a small focused probe, which allow for simultaneous acquisition of incoherent imaging modes such as ADF. 
Focused probe ptychography is performed using the STEM configured as for any atomic resolution incoherent imaging mode. For each probe position, the intensity in the STEM detector plane is recorded on a pixelated detector resulting in a four-dimensional (4D) dataset. Two of these dimensions correspond to the real-space probe position, and two correspond to reciprocal space positions in the convergent beam electron diffraction (CBED) pattern recorded at the detector plane. As shown by Rodenburg and Bates ${ }^{16}$ and Rodenburg et al., ${ }^{17}$ the transmission function of the sample, including the phase shift introduced by light elements, can be retrieved from the 4D STEM data without the need for real or virtual phase plates, as is the case for conventional TEM.

Following the approach of Rodenburg and Bates, ${ }^{16}$ the ptychographic process is described as follows: we denote $M\left(\boldsymbol{K}_{\boldsymbol{f}}, \boldsymbol{R}_{\boldsymbol{p}}\right)$ as the complex amplitude of the electron wave in the diffraction plane, with respect to a scattering vector $\boldsymbol{K}_{f}$ and probe position $\boldsymbol{R}_{\boldsymbol{p}}$. The detector records the intensity in the diffraction plane, which for a thin sample can be written as

$$
\left|M\left(\boldsymbol{K}_{\boldsymbol{f}}, \boldsymbol{R}_{\boldsymbol{p}}\right)\right|^{2}=\left|A\left(\boldsymbol{K}_{\boldsymbol{f}}\right) \exp \left(i 2 \pi \boldsymbol{K}_{\boldsymbol{f}} \cdot \boldsymbol{R}_{\boldsymbol{p}}\right) \otimes_{\boldsymbol{K}_{f}} \Psi\left(\boldsymbol{K}_{\boldsymbol{f}}\right)\right|^{2},
$$

where $A\left(\boldsymbol{K}_{f}\right)$ corresponds to the aperture function of the microscope with a modulus that expresses the size and position of the physical probe-forming aperture and any residual lens aberrations expressed as a phase shift in the argument of $A\left(\boldsymbol{K}_{\boldsymbol{f}}\right), \Psi\left(\boldsymbol{K}_{\boldsymbol{f}}\right)$ is the Fourier transform of the specimen transmission function, and $\psi(\boldsymbol{r})$ is a complex function with an argument that expresses the phase shift due to the sample. In order to retrieve the phase of $\psi(\boldsymbol{r})$, a Fourier transform is performed with respect to $\boldsymbol{R}_{\boldsymbol{p}}$ to obtain

$$
G\left(\boldsymbol{K}_{f}, \boldsymbol{Q}_{p}\right)=A\left(\boldsymbol{K}_{f}\right) A^{*}\left(\boldsymbol{K}_{f}+\boldsymbol{Q}_{\boldsymbol{p}}\right) \otimes_{\boldsymbol{K}_{f}} \Psi\left(\boldsymbol{K}_{f}\right) \Psi^{*}\left(\boldsymbol{K}_{f}-\boldsymbol{Q}_{p}\right) .
$$

$G\left(\boldsymbol{K}_{\boldsymbol{f}}, \boldsymbol{Q}_{p}\right)$ can be thought of as a convolution in the detector plane of a function consisting of the overlap region of two aperture functions separated by the spatial frequency of interest in the image, $Q_{p}$, and a function representing the interference of all pairs of diffracted beams separated by $\boldsymbol{Q}_{\boldsymbol{p}}$.

Once $G\left(\boldsymbol{K}_{\boldsymbol{f}}, \boldsymbol{Q}_{\boldsymbol{p}}\right)$ is obtained, $\psi\left(\boldsymbol{R}_{\boldsymbol{p}}\right)$ can be determined using the single sideband (SSB) method ${ }^{17}$ for weak-phase-objects, or the Wigner distribution deconvolution (WDD) method ${ }^{16}$ for general phase-objects. The advantages of ptychography over other STEM phase imaging methods include super-resolution capabilities, robustness to dynamical scattering, and post-processing aberration correction. ${ }^{10,12,18,19}$ The reader is referred to the literature for further information on ptychographic reconstruction algorithms. ${ }^{16,17,20,21}$

Initially, the slow frame rates $(<100 \mathrm{~Hz})$ and low SNRs of early indirect charged-coupled devices (CCDs) limited the application of ptychographic experiments to beam-stable samples, i.e., amorphous carbon ${ }^{17}$ and Si [110]. ${ }^{18}$ In recent years, the emergence of direct, $\mathrm{kHz}$ rate noise-free fast pixelated detectors (FPDs) has given rise to a plethora of applications, such as nanoscale magnetic domain mapping, ${ }^{22}$ low-dose scanning electron diffraction, ${ }^{23}$ and atomic resolution charge density imaging. ${ }^{24}$ The increase in the available detector frame rates has been particularly advantageous for electron ptychography, with recent applications including super-resolution imaging of nanomaterials, ${ }^{10}$ imaging lithium in novel battery materials, ${ }^{1}$ and comparing DFT calculations of charge transfer with experimental phase reconstructions. ${ }^{25}$ Furthermore, the combination of simultaneous ADF imaging with electron ptychography renders quantitative atomic resolution imaging of both light and heavy elements feasible within a single experiment, for a sufficiently thin sample. ${ }^{26}$

Despite recent detector advancements, 4D STEM imaging experiments remain difficult to perform on beam-sensitive materials. Most modern low-noise integrating CMOS and electron counting detectors can record full-frame individual CBED patterns at $\sim 10^{3} \mathrm{~Hz}$, while more traditional non-pixelated (i.e., $\mathrm{ADF}$ and $\mathrm{DPC}$ ) detectors can operate at speeds faster than $10^{5} \mathrm{~Hz}$. The relatively slow speeds of FPDs make 4D STEM acquisitions more susceptible to scan distortions, sample drift and changes to lens aberrations and ultimately, set a minimum possible dose. For example, with a frame rate of $1 \mathrm{kHz}$ and a probe step size of $0.2 \AA$, a current of $0.5 \mathrm{pA}$ would correspond to an electron dose of $7.7 \times 10^{4}$ e $\AA^{-2}$. For reference, this dose value is $1-2$ orders of magnitude greater than the critical dose previously calculated for MFI-type zeolites. ${ }^{27,28}$ A beam current of several femtoamperes would be needed to image such materials without damaging them, for which it is not practically possible to navigate the sample and align the microscope.

Fortunately, modern FPDs possess a variety of acquisition modes, several of which can operate at faster frame rates. Many CCD and integrated CMOS detectors enable hardware binning and windowing, ${ }^{22}$ increasing the frame rates at the expense of pixel resolution and field of view, respectively. Other electron counting detectors allow the user to increase the detector speed at the expense of dynamic range, i.e., the range of intensity values that can be recorded at each detector pixel. For example, for a 6-bit counting mode, each detector pixel can record between 0 and 63 counts. However, as the bit-depth is decreased, the readout time of the on-chip counters decreases and consequently detector frame rates can be increased significantly. In this study, we investigate the performance of phase reconstructions using $4 \mathrm{D}$ datasets acquired with a counter depth of 1-bit (i.e., which can record either 0 or 1) at a frame rate of $12.5 \mathrm{kHz}$.

The 4D STEM datasets were obtained from an aluminosilicate zeolite sample (ZSM-5) using a Quantum Detectors MerlinEM system (see supplementary material Sec. S1) installed on a JEM ARM300CF. An acceleration voltage of $300 \mathrm{kV}$ was chosen which optimized the electron-optical alignment at low beam currents on the instrument used and minimized beam damage of the specimen (see supplementary material Sec. S2). For this experiment, $256 \times 256 \mathrm{CBED}$ patterns, each consisting of $256 \times 256$ pixels, were recorded using the 1-bit counting mode at $12.5 \mathrm{kHz}$ (see supplementary material Sec. S3 for more details). To minimize the beam current at the sample, the probe semi-convergence angle was set to $7.6 \mathrm{mrad}$. This value was sufficient to resolve the ZSM-5 framework, while reducing noise from unwanted higher spatial frequencies.

A sequence of CBED patterns from the acquired $4 \mathrm{D}$ dataset is shown in Fig. 1(a) (Multimedia view), which have an average of 272 counts per frame and the position-averaged CBED pattern is displayed in Fig. 1(b). Dead pixels, which comprised $0.05 \%$ of the bright-field disk, had no noticeable effect on the reconstruction (see supplementary material Sec. S3). The beam current was measured as $0.3 \mathrm{pA}$ using a Faraday

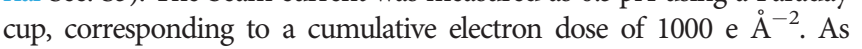
shown in Fig. 1(a), this beam current was sufficiently low such that each electron event was isolated within the CBED patterns.

Recording 4D STEM data with a 1-bit counter depth is advantageous for several reasons. First, for a frame rate of $1 \mathrm{kHz}$, a $256 \times 256$ 


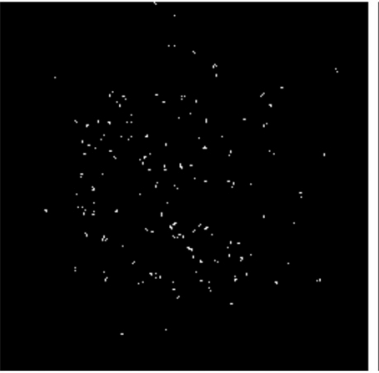

(a)

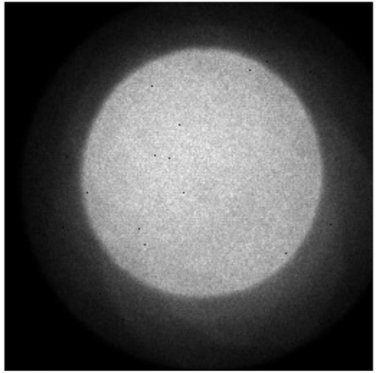

(b)
FIG. 1. (a) CBED frame sequence and (b) position-averaged CBED pattern for ZSM-5. Multimedia view: https://doi.org/10.1063/1.5143213.1

probe position scan would take over one minute to acquire. This is sufficiently long for the specimen or low order aberration drift to have a detrimental effect on the acquired data. At $12.5 \mathrm{kHz}$, a $256 \times 256$ probe position scan takes just over $10 \mathrm{~s}$ and drift rates are significantly reduced. In addition, faster frame rates enable data acquisition at higher, more optically favorable probe currents for a given electron dose.

Figure 2 shows (a) the modulus and (b) phase of $G\left(\boldsymbol{K}_{\boldsymbol{f}}, \boldsymbol{Q}_{\boldsymbol{p}}\right)$ for an example $Q_{p}=\mathbf{4 . 9} \mathrm{mrad}$. Although each individual CBED pattern is sparse, the phase of $G\left(\boldsymbol{K}_{\boldsymbol{f}}, \boldsymbol{Q}_{\boldsymbol{p}}\right)$ is still transferred with a high SNR showing that the variation with respect to the probe position of the statistical likelihood of electron arrival in a particular region of the detector is sufficient to enable phase detection. One advantage of SSB ptychography is that, because only the regions of constructive interference are used for the reconstruction process, much of the unwanted noise is rejected.

The results of the binary 4D STEM experiment on ZSM-5 [010] are shown in Fig. 3 (see supplementary material Sec. S4 for analysis workflow). To demonstrate the versatility of the data, two independent phase reconstruction methods were performed: integrated center-ofmass (iCoM) ${ }^{9}$ (i.e., iDPC using a pixelated detector) and SSB ptychography. The simultaneously obtained ADF image shown in Fig. 3(a) reveals the zeolite structure, but with a poor SNR. The iCoM and SSB reconstructions are shown in Figs. 3(b) and 3(c), respectively, and both show atomically resolved contrast. The SSB reconstruction was normalized with respect to the noise ${ }^{14}$ to broaden the window of information transfer. The power spectrum of the SSB phase reconstruction is shown in Fig. 3(d), where the $(10,0,0)$ reflection is resolved, corresponding to a spacing of $2.01 \AA$.

To test the efficiency of phase imaging techniques at a low dose using binary 4D STEM, a second dataset was acquired using a beam

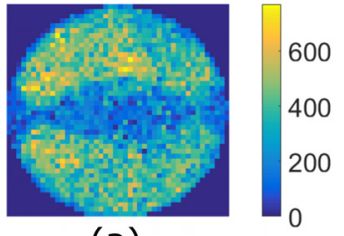

(a)

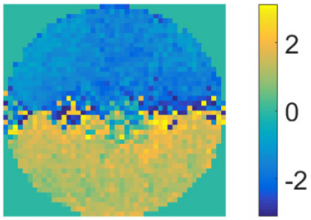

(b)
FIG. 2. (a) Modulus (arb. units) and (b) phase (radians) of $G\left(K_{f}, 4.9 \mathrm{mrad}\right)$ for ZSM-5.

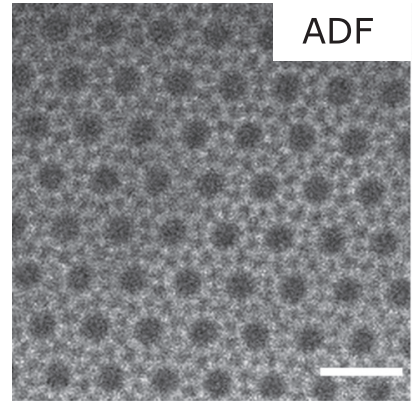

(a)

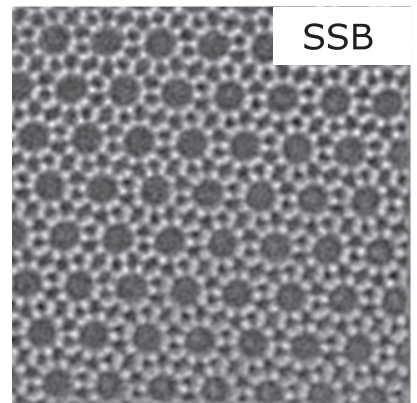

(c)

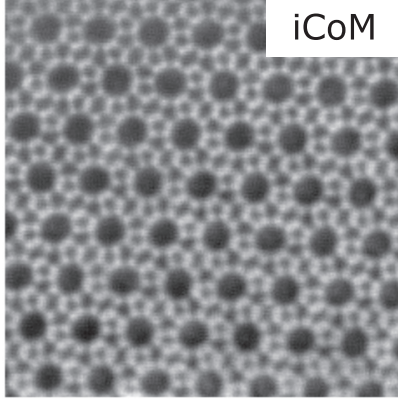

(b)

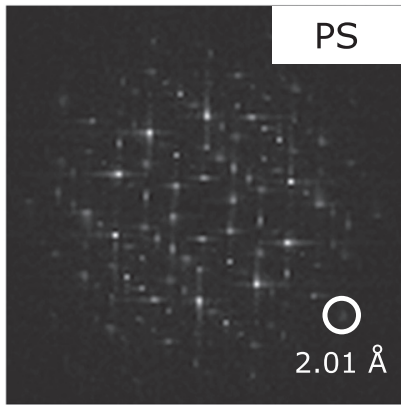

(d)
FIG. 3. (a) ADF image, (b) iCoM reconstruction, and (c) SSB reconstruction for ZSM-5. Scale bar: $2 \mathrm{~nm}$. The power spectrum (PS) of (c) is shown in (d). The $(10,0,0)$ reflection, corresponding to a spacing of $2.01 \AA$, is marked with a white circle.

current of $0.06 \mathrm{pA}$, such that the cumulative electron dose was $200 \mathrm{e}$ $\AA^{-2}$. An example CBED pattern sequence for the data is shown in Fig. 4(a) (Multimedia view). In the static figure showing one acquired CBED pattern, there are a total of 19 detector counts, corresponding to 9 electron events (see supplementary material Sec. S1). The iCoM and SSB reconstructions are shown in Figs. 4(b) and 4(c), respectively. In this case, the ZSM-5 region of interest is wedge-like, with the maximum thickness in the top left of the image. In the thicker region, the zeolite structure is resolved, but in the thinner region, there is insufficient scattering to provide significant sample information.

Conversely, for high electron doses $\left(10^{6}\right.$ e $\left.\AA^{-2}\right)$, a 1-bit counting mode using current detectors leads to saturation. However, with stateof-the-art FPDs reaching speeds of $0.1 \mathrm{MHz},{ }^{29}$ future binary 4D STEM

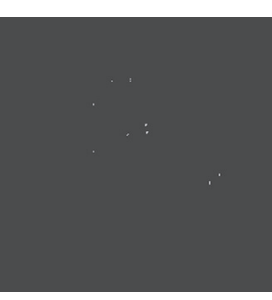

(a)

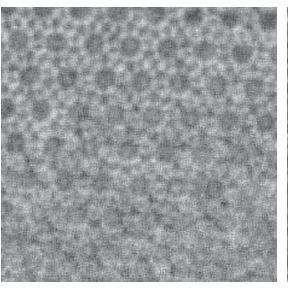

(b)

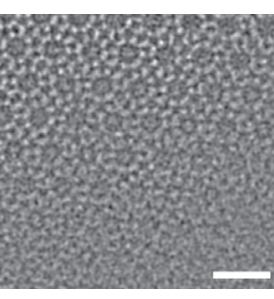

(c)
FIG. 4. (a) Example CBED pattern sequence, (b) iCoM reconstruction, and (c) SSB reconstruction for ZSM- 5 using a cumulative electron dose of $200 \mathrm{e} \AA^{-2}$. The scale bar for the phase reconstructions is $2 \mathrm{~nm}$. Multimedia view: https://doi.org/10.1063/ 1.5143213 .2 
experiments may not require any change in the probe current between sample screening and acquisition. This will be essential for imaging light-element or beam-sensitive nanomaterials, for which large probe convergence angles and minimal high-order aberrations are necessary.

In conclusion, we have demonstrated the application of binary $4 \mathrm{D}$ STEM data to post-processing phase reconstruction techniques. The 1-bit counting mode on a MerlinEM system can record 10 times faster than 12-bit counting mode, which is within one order of typical conventional ADF imaging speeds. Importantly, the results demonstrate that FPDs do not need a high dynamic range to provide highquality phase reconstructions and that faster detectors should enable high throughput, low dose 4D STEM phase imaging in the near future. Furthermore, multi-frame acquisition combined with image registration techniques is increasingly used to increase the SNR of STEM images and faster scanning will allow this approach to also be used with pixelated detectors. ${ }^{30,31}$

See the supplementary material for further details of the MerlinEM system, experimental setup, and data analysis workflow.

The authors acknowledge funding from the EPSRC (Grant No. EP/M010708/1) and JEOL (UK) Ltd. We thank the Diamond Light Source for access and support in the use of the electron Physical Science Imaging Centre (Instrument E02, Proposal Nos. MG20431 and MG22317) that contributed to the results presented here.

\section{REFERENCES}

${ }^{1}$ J. G. Lozano, G. T. Martinez, L. Jin, P. D. Nellist, and P. G. Bruce, Nano Lett. 18, 6850-6855 (2018).

${ }^{2}$ M. U. Rothmann, W. Li, J. Etheridge, and Y.-B. Cheng, Adv. Energy Mater. 7, 1700912 (2017)

${ }^{3}$ D. Zhang, Y. Zhu, L. Liu, X. Ying, C. Hsiung, R. Sougrat, K. Li, and Y. Han, Science 359, 675-679 (2018).

${ }^{4}$ L. Liu, Z. Chen, J. Wang, D. Zhang, Y. Zhu, S. Ling, K. Huang, Y. Belmabkhout, A. Karim, Y. Zhang, B. Slater, E. Mohamed, and Y. Han, Nat. Chem. 11, 622-628 (2019).

${ }^{5}$ O. Scherzer, J. Appl. Phys. 20, 20-29 (1949).

${ }^{6}$ F. Zernike, Physica 9, 974-986 (1942).

${ }^{7}$ A. Varambhia, L. Jones, A. De Backer, V. Fauske, S. Van Aert, D. Ozkaya, S. Lozano-Perez, and P. Nellist, in European Microscopy Congress 2016: Proceedings (2016), pp. 537-538.
${ }^{8}$ N. Shibata, S. D. Findlay, Y. Kohno, H. Sawada, Y. Kondo, and Y. Ikuhara, Nat. Phys. 8, 611-615 (2012).

${ }^{9}$ I. Lazić, E. G. T. Bosch, and S. Lazar, Ultramicroscopy 160, 265--280 (2016).

${ }^{10}$ Y. Jiang, Z. Chen, Y. Han, P. Deb, H. Gao, S. Xie, P. Purohit, M. W. Tate, J. Park, S. M. Gruner, V. Elser, and D. A. Muller, Nature 559, 343-349 (2018).

${ }^{11}$ M. J. Humphry, B. Kraus, A. C. Hurst, A. M. Maiden, and J. M. Rodenburg, Nat. Commun. 3, 730 (2012).

${ }^{12}$ H. Yang, R. N. Rutte, L. Jones, M. Simson, R. Sagawa, H. Ryll, M. Huth, T. J. Pennycook, M. L. H. Green, H. Soltau, Y. Kondo, B. G. Davis, and P. D. Nellist, Nat. Commun. 7, 12532 (2016).

${ }^{13}$ S. D. Findlay, R. Huang, R. Ishikawa, N. Shibata, and Y. Ikuhara, Microscopy 66, 3-14 (2017).

${ }^{14}$ T. Seki, Y. Ikuhara, and N. Shibata, Ultramicroscopy 193, 118-125 (2018).

${ }^{15}$ J. Song, C. S. Allen, S. Gao, C. Huang, H. Sawada, X. Pan, J. Warner, P. Wang, and A. I. Kirkland, Sci. Rep. 9, 3919 (2019).

${ }^{16}$ J. M. Rodenburg and R. H. T. Bates, Philos. Trans. R. Soc., A 339, 521-553 (1992).

17J. M. Rodenburg, B. C. McCallum, and P. D. Nellist, Ultramicroscopy 48, 304-314 (1993).

${ }^{18}$ P. D. Nellist, B. C. McCallum, and J. M. Rodenburg, Nature 374, 630-632 (1995).

${ }^{19}$ T. Plamann and J. M. Rodenburg, Acta Crystallogr., Sect. A 54, 61-73 (1998).

${ }^{20}$ J. M. Rodenburg and H. M. L. Faulkner, Appl. Phys. Lett. 85, 4795-4797 (2004).

${ }^{21}$ A. M. Maiden and J. M. Rodenburg, Ultramicroscopy 109, 1256-1262 (2009).

${ }^{22}$ H. Ryll, M. Simson, R. Hartmann, P. Holl, M. Huth, S. Ihle, Y. Kondo, P. Kotula, A. Liebel, K. Müller-Caspary, A. Rosenauer, R. Sagawa, J. Schmidt, H. Soltau, and L. Strüder, J. Instrum. 11, P04006 (2016).

${ }^{23}$ D. N. Johnstone, C. S. Allen, M. Danaie, R. C. B. Copley, J. Brum, A. I. Kirkland, and P. A. Midgley, Microscopy Microanal. 25(S2), 1746-1747 (2019).

${ }^{24}$ W. Gao, C. Addiego, H. Wang, X. Yan, Y. Hou, D. Ji, C. Heikes, Y. Zhang, L. Li, H. Huyan, T. Blum, T. Aoki, Y. Nie, D. G. Schlom, R. Wu, and X. Pan, Nature 575, 480-484 (2019).

${ }^{25}$ G. T. Martinez, T. C. Naginey, L. Jones, C. M. O’Leary, T. J. Pennycook, R. J. Nicholls, J. R. Yates, and P. D. Nellist, arXiv:1907.12974v1 (2019).

${ }^{26}$ E. Liberti, G. T. Martinez, C. M. O’Leary, P. D. Nellist, and A. I. Kirkland, Microscopy Microanal. 25(S2), 8-9 (2019).

${ }^{27}$ M. Pan and P. A. Crozier, Ultramicroscopy 48, 332-340 (1993).

${ }^{28}$ K. Yoshida and Y. Sasaki, J. Electron Microsc. 62, 369-375 (2013).

${ }^{29}$ J. Ciston, I. J. Johnson, B. R. Draney, P. Ercius, E. Fong, A. Goldschmidt, J. M. Joseph, J. R. Lee, A. Mueller, C. Ophus, A. Selvarajan, D. E. Skinner, T. Stezelberger, C. S. Tindall, A. M. Minor, and P. Denes, Microscopy Microanal. 25(S2), 1930-1931 (2019).

${ }^{30} \mathrm{X}$. Sang and J. M. LeBeau, Ultramicroscopy 138, 28-35 (2014).

${ }^{31}$ L. Jones, H. Yang, T. J. Pennycook, M. S. J. Marshall, S. Van Aert, N. D. Browning, M. R. Castell, and P. D. Nellist, Adv. Struct. Chem. Imaging 1, 8 (2015). 